\title{
Associação de aves a agrupamentos de bambu na porção Sul da Mata Atlântica, Londrina, Estado do Paraná, Brasil
}

\author{
Cássius Ricardo Santana ${ }^{1,2,}$ \& Luiz dos Anjos ${ }^{1}$ \\ ${ }^{1}$ Programa de Pós-Graduação em Ciências Biológicas, Departamento de Biologia Animal e Vegetal, \\ Universidade Estadual de Londrina - UEL, \\ CP 6001, CEP 86051-990, Londrina, PR, Brasil \\ ${ }^{2}$ Autor para correspondência: Cássius Ricardo Santana, e-mail: cassius.santana@gmail.com
}

SANTANA, C.R. \& ANJOS, L. On the association of birds to bamboo stands in Southern brazilian Atlantic Forest. Biota Neotrop. 10(2): http://www.biotaneotropica.org.br/v10n2/en/abstract?article+bn00510022010.

\begin{abstract}
Association of birds to bamboo stands in southern brazilian Atlantic Forest, Londrina, Parana State, Brazil. Bamboo stands have been considered relatively important for a high number of bird species, specially in Amazonia. In the present study variations on the abundance of bamboo associated birds were evaluated in areas with different availability of bamboo Chusquea sp. present inside native forest in the southern portion of the Atlantic Forest, Parana State. Point counts were performed during 25 days in five transects, three in bamboo stands areas and two where this microhabitat was absent; the transects differ in the availability of bamboo. The study areas were the Mata dos Godoy State Park and an adjacent forest fragment, which together sum a total area larger than 2,000 ha, in northern Parana State, southern Brazil. Nineteen bird species were analyzed and only five of them were recorded in the transects where the bamboo was absent. Five bird species decreased significantly their abundances according the decreasing in the availability of bamboo (G-test, $\mathrm{P}<0.01$ ). None species had higher abundance in the transects where the bamboo was absent. Differently of Amazonia, bamboo stands in the southern portion of the Brazilian Atlantic forest are not extensive areas; actually, this type of microhabitat is more disperse throughout the understory. Even so, this microhabitat may be important for conservation of certain bird species in the Atlantic forest.
\end{abstract}

Keywords: birds, bamboo, Chusquea, Atlantic Forest.

SANTANA, C.R. \& ANJOS, L. Associação de aves a agrupamentos de bambu na porção Sul da Mata Atlântica, Londrina, Estado do Paraná, Brasil. Biota Neotrop. 10(2): http://www.biotaneotropica.org.br/ v10n2/pt/abstract?article+bn00510022010.

Resumo: Agrupamentos de bambu têm sido considerados relativamente importantes para um grande número de espécies de aves, principalmente na Amazônia. No presente estudo avaliamos as variações na abundância de aves associadas ao bambu Chusquea sp. em áreas com diferentes disponibilidades deste microhábitat no interior de floresta nativa, na porção sul da Mata Atlântica, Estado do Paraná. Amostragens por ponto-de-escuta foram realizadas durante 25 dias em cinco trilhas, três em áreas com agrupamentos de bambu e duas em áreas onde este microhábitat estava ausente; as trilhas diferiram na disponibilidade de bambu. As áreas de estudos foram o Parque Estadual Mata dos Godoy e um fragmento florestal adjacente que, juntos, formam uma área maior que 2.000 ha no Norte do Estado do Paraná, Sul do Brasil. Das dezenove espécies de aves analisadas, apenas cinco foram registradas nas trilhas onde não havia bambu. Cinco espécies diminuíram significativamente suas abundâncias de acordo com a disponibilidade decrescente de aglomerados de bambu (teste- $\mathrm{G}, \mathrm{P}<0,01$ ). Nenhuma espécie de ave apresentou maior abundância nas trilhas onde o bambu estava ausente. Diferente da Amazônia, agrupamentos de bambu na porção sul da Mata Atlântica brasileira não formam áreas extensas, sendo encontrados mais esparsos pelo sub-bosque. Ainda assim, este microhábitat pode ser importante para a conservação de certas espécies de aves na Mata Atlântica.

Palavras-chave: aves, bambu, Chusquea, Mata Atlântica. 


\section{Introdução}

O bambu aparece como um elemento vegetal importante na composição de muitas florestas tropicais e temperadas, como as presentes na Ásia, África e América do Sul (Janzen 1976, Veblen 1982, Stotz et al. 1996). Os bambus são reconhecidos por crescerem em agregados monotípicos, com colmos de tamanho, espessura e flexibilidade variáveis que podem se entrelaçar, formando um ambiente denso com um estrato inferior de folhas anatomicamente semelhantes (Kratter 1997, Ohrnberger 1999, Bodrati \& Cockle 2006). Essas plantas também são caracterizadas por apresentarem floração sincronizada após décadas de crescimento vegetativo, sucedida de uma massiva produção de sementes e posterior degeneração que pode atingir grandes áreas (Janzen 1976). Devido a essas características, os bambuzais tornam-se microhábitats particulares, dentro dos ecossistemas florestais, cuja dinâmica afeta a sobrevivência de aves e outros animais (Olmos et al. 1993, Olmos 1996, Jaksic \& Lima 2003). Além da disponibilidade de alimento para várias espécies durante a frutificação, os agrupamentos de bambu fornecem abrigo e, principalmente, presas em potencial para aves insetívoras (Olmos 1996, Kratter 1997, Silveira 1999, Reid et al. 2004, Vasconcelos et al. 2005).

A ocorrência de associação de aves com bambu no Neotrópico tem sido documentada principalmente na Floresta Amazônica onde os bambus formam extensos agrupamentos (Parker 1982, Pierpoint \& Fitzpatrick 1983, Kratter 1997, Kratter \& Parker 1997, Parker et al. 1997, Aleixo et al. 2000, Lloyd 2004). Kratter (1997) identificou pelo menos 32 espécies de aves associadas a esse microhábitat em um estudo desenvolvido no sudeste do Peru.

A Mata Atlântica também é um centro de diversidade de espécies de bambu (Judziewicz et al. 1999). Neste bioma, porém, os agrupamentos de bambu tendem a cobrir área menor, quando comparados aos encontrados na Amazônia. Na porção sul da Mata Atlântica, os agrupamentos de bambu, frequentemente, ocupam clareiras e áreas de crescimento secundário, especialmente na mata ripária ou de encosta, onde se distribuem de forma agregada em pequenos agrupamentos relativamente esparsos entre si. Apesar de várias espécies de aves terem sido registradas como associadas aos agrupamentos de bambu na Mata Atlântica, não é claro o nível dessa associação de acordo com a disponibilidade deste microhábitat (Rodrigues et al. 1994, Stotz et al. 1996, Goerck 1999, Leme 2001, Lopes et al. 2006, Rajão \& Cerqueira 2006, Volpato et al. 2006, Antunes 2007).

No presente estudo avaliamos variações na abundância de espécies de aves associadas ao bambu Chusquea sp. em áreas com diferentes disponibilidades deste vegetal em floresta nativa. Nossa intenção foi testar a hipótese de que as aves apresentam abundâncias diferentes de acordo com a disponibilidade do recurso vegetal avaliado em remanescente de Mata Atlântica na região de Londrina, norte do estado do Paraná.

\section{Materiais e Métodos}

\section{1. Área de estudo}

O estudo foi desenvolvido no Parque Estadual Mata dos Godoy (PG, 656 ha; $23^{\circ} 27^{\prime} \mathrm{S}$ e $51^{\circ} 14^{\prime} \mathrm{O}$ ) e em um fragmento florestal adjacente (FG, 285 ha), ligado ao parque por um corredor florestal de aproximadamente $100 \mathrm{~m}$ de largura. Juntas, as duas áreas e outras adjacentes somam mais de 2.000 ha e localizam-se nas proximidades do município de Londrina, estado do Paraná, sul do Brasil. Cerca de metade da área do Parque é plana $(600 \mathrm{~m})$, sendo o restante inclinado, atingindo a porção mais baixa no limite sul $(470 \mathrm{~m})$, onde está o Ribeirão dos Apertados. A floresta (Figura 1) se mostra claramente

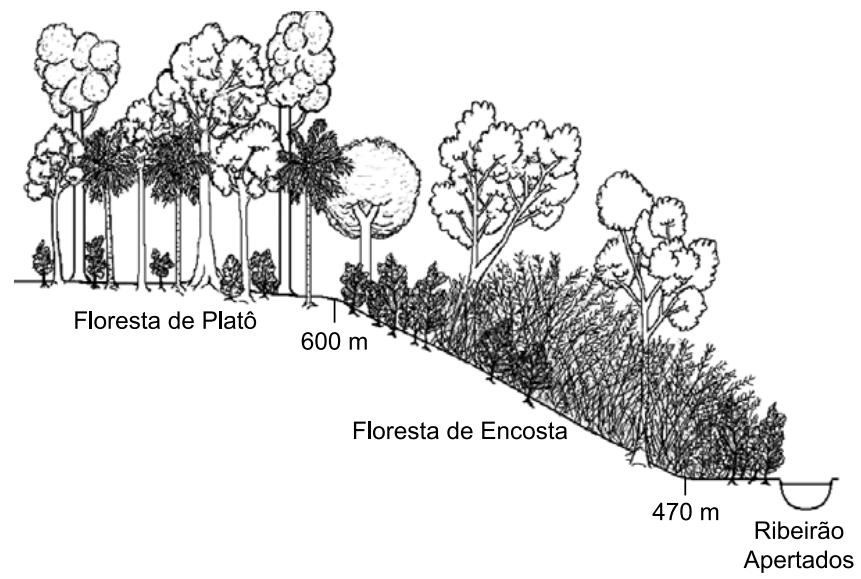

Figura 1. Perfil esquemático representando os dois tipos de floresta presentes no Parque Estadual Mata dos Godoy, município de Londrina, Paraná, Brasil.

Figure 1. Schematic drawing showing the two types of Forest observed in the Parque Estadual Mata dos Godoy, Londrina, Parana, Brazil.

diferente na porção plana em relação à porção inclinada (de encosta). A floresta da porção plana, denominada aqui como floresta de platô, apresenta um estrato superior denso, com dominância de espécies como Cabralea canjerana (Meliaceae) e Euterpe edulis (Arecaceae), e um estrato emergente composta especialmente por Aspidosperma polyneuron (Apocynaceae) e Sloanea monosperma (Elaeocarpaceae). Os estratos inferior e médio são mais rarefeitos, pouco iluminados e compostos por arbustos esparsos e dominados por algumas árvores de pequeno porte, como Eugenia verrucosa (Myrtaceae) e Sorocea bonplandii (Moraceae) (Bianchini et al. 2001). Já na porção inclinada, a floresta, aqui denominada floresta de encosta, apresenta o estrato superior mais rarefeito, com poucas árvores emergentes, como Chrysophyllum gonocarpum (Sapotaceae) e Campomanesia xanthocarpha (Myrtaceae), enquanto que os estratos inferior e médio são dominados por grande quantidade de pequenas árvores como Nectandra megapotamica (Lauraceae), Lonchocarpus muehlbergianus (Fabaceae) e Eugenia verrucosa (Myrtaceae). Nesta área, é comum a queda das árvores mais altas e conseqüente formação de clareiras onde predominam a herbácea Celtis iguanaea (Ulmaceae) e o bambu Chusquea sp. (Poaceae) (Bianchini et al. 2001). O fragmento de 285 ha possui relevo inclinado, sendo composto quase que totalmente pela floresta de encosta.

Foram estabelecidas quatro trilhas distantes pelo menos $300 \mathrm{~m}$ entre si, em diferentes pontos do PG e uma trilha em FG (Figura 2). Na floresta de platô do PG foram estabelecidas duas trilhas situadas perpendicularmente entre si e caracterizadas por não possuírem bambu ao longo de sua extensão, referidas como SB1 e SB2 ( $\mathrm{SB}=$ sem bambu). Na floresta de encosta foram demarcadas outras três trilhas com agrupamentos de bambu, denominadas $\mathrm{CB} 1, \mathrm{CB} 2 \mathrm{e}$ CB3 (CB = com bambu). A disponibilidade de bambu foi avaliada por meio de estimativas visuais de cobertura em um raio de $10 \mathrm{~m}$ a partir de seis pontos aleatórios estabelecidos em cada trilha. As três trilhas com bambu diferiram quanto à disponibilidade desse recurso (Figura 3). O agrupamento da trilha CB1 caracterizou-se como quase puro de Chusquea, ocupou uma clareira de cerca de 8 ha ao final de um declive acentuado e apresentou uma porcentagem média de $92 \%$ de cobertura de bambu. A trilha CB2 continha um agrupamento não puro de Chusquea (média de $68 \%$ de cobertura), sem a presença de grandes clareiras, onde o bambu encontrava-se associado a outras espécies 


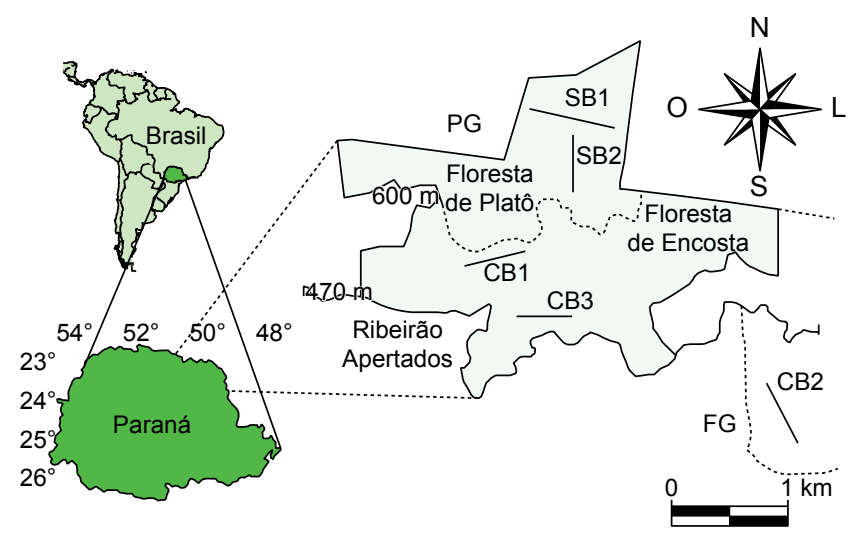

Figura 2. Localização das cinco trilhas amostradas no Parque Estadual Mata dos Godoy e no fragmento FG (linha tracejada), município de Londrina, Paraná, Brasil. A linha pontuada indica a divisão, por altitude, dos dois tipos de floresta do Parque. Modificado de Anjos et al. (2007b).

Figure 2. Location of the five transects sampled in the Parque Estadual Mata dos Godoy and in the fragment FG (spaced line), Londrina, Parana, Brazil. The line with points shows the division, by elevation, of the two types of forest. Adapted from Anjos et al. (2007b).
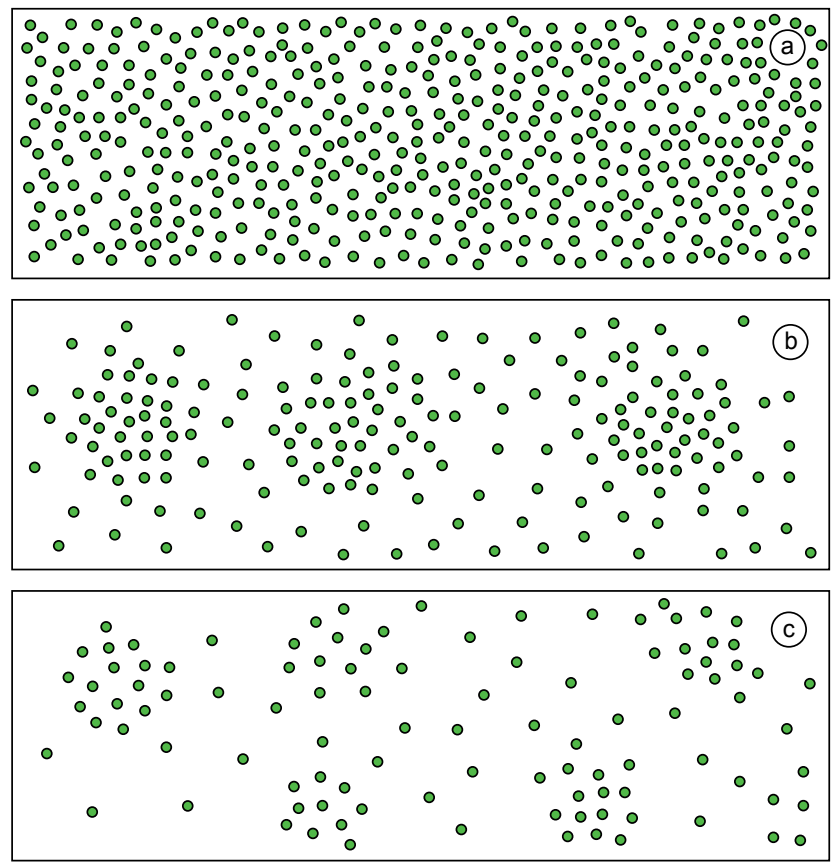

Figura 3. Representação esquemática da distribuição de bambu nas trilhas a) $\mathrm{CB} 1$, b) CB2 e c) CB3.

Figure 3. Schematic drawing showing bamboo distribution along the transects a) $\mathrm{CB} 1$, b) $\mathrm{CB} 2$ and c) $\mathrm{CB} 3$.

vegetais, tais como Cecropia sp. (Cecropiaceae), Eugenia verrucosa (Myrtaceae), Euterpe edullis (Arecaceae) e Nectandra megapotamica (Lauraceae). O agrupamento da trilha CB3 foi similar ao da trilha CB2; porém, no caso da trilha $\mathrm{CB} 3$ os agrupamentos de bambu foram de menor tamanho e mais isolados, distribuídos ao longo da margem do Ribeirão dos Apertados e com seus colmos dispersos entre as espécies mencionadas para a floresta de encosta do PG, o que resultou em uma menor percentagem média de Chusquea (37\%).

\section{Abundância das espécies de aves}

Um levantamento da literatura (Stotz et al. 1996, Zimmer \& Isler 2003, Fitzpatrick 2004, Lopes et al. 2006), bem como observações prévias em campo, permitiram a seleção de 19 espécies associadas ao bambu presentes na área de estudo. O método de amostragem por pontos de escuta (Blondel et al. 1970, Vielliard \& Silva 1990, Anjos 2007a) foi aplicado nas cinco trilhas previamente descritas, o que possibilitou a obtenção do Índice Pontual de Abundância (IPA) para cada espécie de ave associada a bambu. Em cada uma das trilhas foram estabelecidos seis pontos, distantes $120 \mathrm{~m}$ entre si. Em cada ponto, o tempo de permanência foi de 15 minutos, com tempo equivalente para deslocamento entre eles. Foram realizados cinco dias alternados de amostragem em cada trilha entre os meses de setembro e dezembro de 2007, totalizando 25 dias de amostragem. As amostragens foram iniciadas em média duas horas após o nascer do sol, em torno das 07:30 horas, e estenderam-se até aproximadamente três horas após seu início. Cada casal ou bando de cada espécie registrado durante a vocalização foi considerado como um contato, sendo o IPA obtido pela divisão do número total de contatos pelo número total de pontos amostrados em cada trilha. A classificação taxonômica das aves seguiu o Comitê Brasileiro de Registros Ornitológicos (CBRO 2009).

\section{Testes estatísticos}

O teste G, com fator de correção de William (Fowler \& Cohen, 1995), foi utilizado para avaliar diferenças nos valores de IPA (x100) das espécies registradas entre as trilhas com disponibilidade de bambu. Os dados de abundância foram avaliados por uma Análise de Correspondência Destendenciada (ACD), interpretada através da adoção dos eixos 1 e 2 e da aplicação uma ANOVA unifatorial, seguida de Teste de Tukey, sobre os escores retidos para esses eixos.

\section{Resultados}

As 19 espécies de aves selecionadas pelo presente estudo foram registradas em pelo menos uma das trilhas com bambu (CB1, CB2 e CB3), sendo que cinco delas também foram registradas naquelas sem bambu (SB1 E SB2). Para as trilhas com bambu, os totais de espécies registrados foram similares (14 espécies em CB1, 16 em CB2 e 15 em CB3) (Tabela 1).

Quatro espécies foram exclusivamente registradas em apenas uma trilha com bambu: três (Corythopis delalandi, Platyrhinchus mystaceus e Cnemotriccus fuscatus) em CB3 e uma (Drymophila rubricollis) em CB2. Oito espécies que ocorreram nas trilhas com bambu apresentaram maior abundância em apenas uma delas (Teste G; $\mathrm{P}<0,01$ ): seis espécies em CB1 (Mackenziaena severa, Drymophila malura, Pyriglena leucoptera, Myiornis auricularis, Poecilotriccus plumbeiceps e Capsiempis flaveola) e duas (Synallaxis cinerascens e Hemitriccus diops) em CB2. As outras sete espécies tiveram abundâncias similares em duas trilhas: cinco (Conopophaga lineata, Hylopezus naterreri, Synallaxis ruficapilla, Cranioleuca obsoleta e Heliobletus contaminatus) tiveram maior IPA em CB1/CB2 e as outras duas (Automolus leucophthalmus e Lathrotriccus euleri) em CB2/CB3. Nenhuma espécie apresentou maior IPA nas trilhas sem bambu (Tabela 1).

Na Análise de Correspondência Destendenciada (ACD), baseada na abundância das espécies, os eixos 1 e 2 foram responsáveis por 29 e $12 \%$ da variação, respectivamente. O eixo 1 agrupou as trilhas que possuem agrupamentos de bambu (Figura 4), principalmente CB1 e CB2 (ANOVA/Teste de Tukey p <0,05). Estas trilhas foram agrupadas especialmente em função de Drymophila rubricollis, Hylopezus nattereri e Synallaxis cinerascens (Figura 5). 


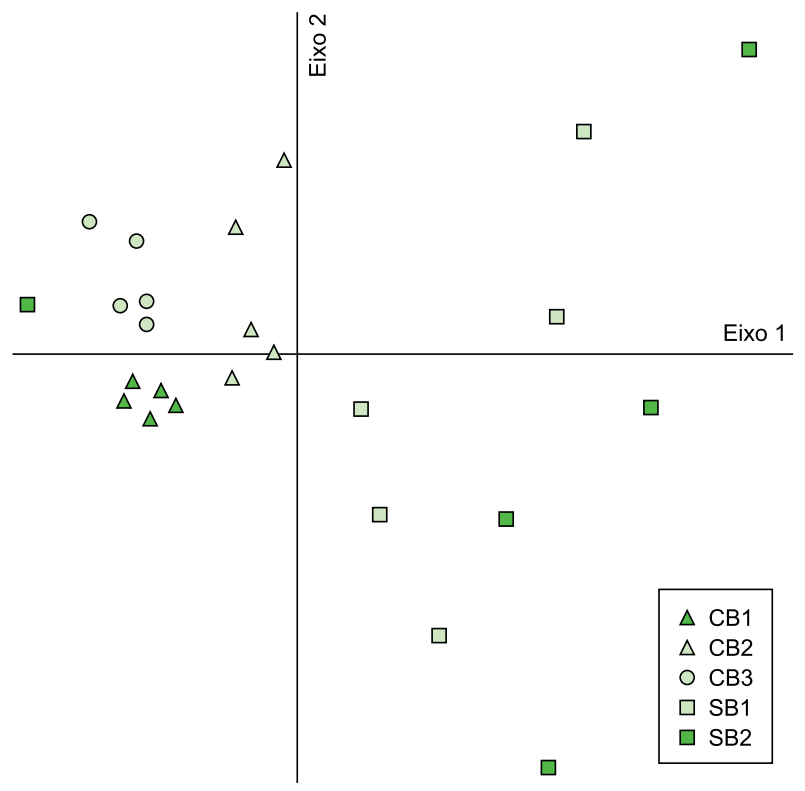

Figura 4. Ordenação por Análise de Correspondência Destendenciada (ACD) dos dias de amostragem em cada trilha utilizando os valores de IPA diários para cada espécie no remanescente do Parque Estadual Mata dos Godoy, município de Londrina, Paraná.

Figure 4. Ordenation by Detrended Correspondece Analysis (DCA) of the two days of sampling in each one of the transects using the daily IPA values for each species in the Parque Estadual Mata dos Godoy, Londrina, Parana.

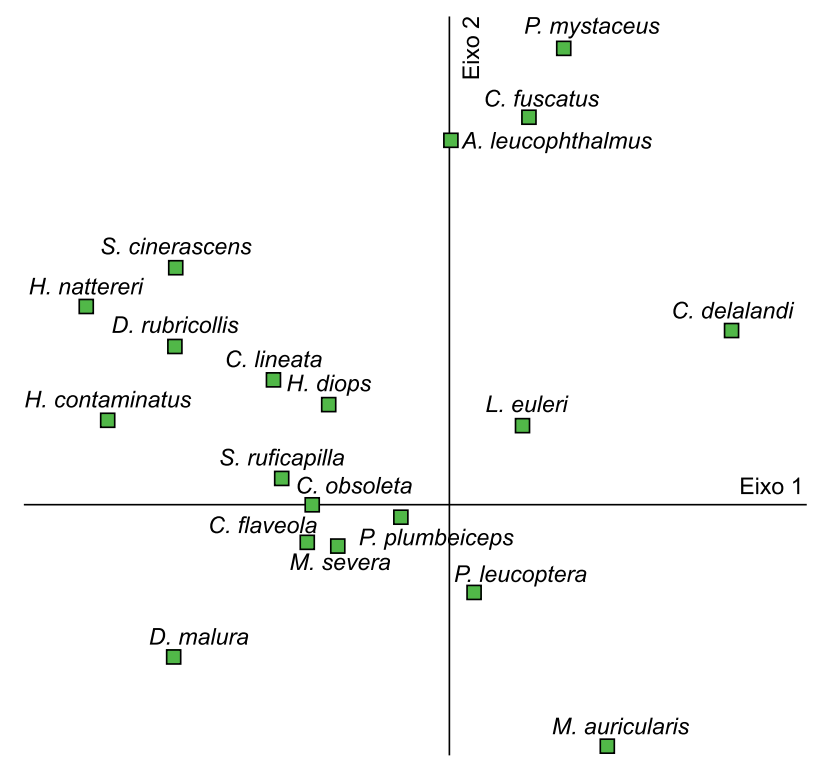

Figura 5. Ordenação por Análise de Correspondência Destendenciada (ACD) das espécies utilizando os valores de IPA diários em cada trilha no remanescente do Parque Estadual Mata dos Godoy, município de Londrina, Paraná.

Figure 5. Ordenation by Detrended Correspondece Analysis (DCA) of the species using the daily IPA values for each transect in the Parque Estadual Mata dos Godoy, Londrina, Parana.

Tabela 1. Índice Pontual de Abundância (IPA) das espécies de aves associadas ao bambu Chusquea sp. em cada trilha amostrada no remanescente do Parque Estadual Mata dos Godoy, norte do Paraná. Letras diferentes sobrescritas nos valores indicam existência de diferença significativa detectada por meio de teste $\mathrm{G}(\mathrm{p}<0,01)$. A classificação taxonômica das aves seguiu o Comitê Brasileiro de Registros Ornitológicos (CBRO 2009).

Table 1. Ponctual Index of Abundance (IPA) of bamboo Chusquea sp. associated birds in each of the trails sampled in the Parque Estadual Mata dos Godoy, northern Parana State. The different letters beside the values point the existence of significative difference between these values, using the G-test $(\mathrm{p}<0,01)$. The taxonomic classification is according to Comitê Brasileiro de Registros Ornitológicos (CBRO 2009).

\begin{tabular}{|c|c|c|c|c|c|}
\hline \multirow[t]{3}{*}{ Famílias e espécies } & \multicolumn{5}{|c|}{$\begin{array}{r}\text { Trilhas } \\
\end{array}$} \\
\hline & \multicolumn{3}{|c|}{ Com bambu } & \multicolumn{2}{|c|}{ Sem bambu } \\
\hline & CB1 & CB2 & CB3 & SB1 & SB2 \\
\hline \multicolumn{6}{|l|}{ THAMNOPHILIDAE } \\
\hline Mackenziaena severa (Lichtenstein, 1823) & $1,70^{\mathrm{a}}$ & $0,87^{\mathrm{b}}$ & $0,67^{\mathrm{b}}$ & - & - \\
\hline Drymophila rubricollis (Bertoni, 1901) & - & 0,03 & - & - & - \\
\hline Drymophila malura (Temminck, 1825$)$ & $0,53^{\mathrm{a}}$ & $0,10^{\mathrm{b}}$ & - & - & - \\
\hline Pyriglena leucoptera (Vieillot, 1818) & $1,30^{\mathrm{a}}$ & $0,57^{\mathrm{b}}$ & $0,67^{b}$ & $0,20^{\mathrm{c}}$ & $0,07^{\mathrm{d}}$ \\
\hline \multicolumn{6}{|l|}{ CONOPOPHAGIDAE } \\
\hline Conopophaga lineata (Wied, 1831) & $0,27^{\mathrm{a}}$ & $0,30^{\mathrm{a}}$ & $0,10^{\mathrm{b}}$ & - & - \\
\hline \multicolumn{6}{|l|}{ GRALLARIIDAE } \\
\hline Hylopezus ochroleucus (Wied, 1831) & $0,07^{\mathrm{a}}$ & $0,13^{\mathrm{a}}$ & - & - & - \\
\hline \multicolumn{6}{|l|}{ FURNARIIDAE } \\
\hline Synallaxis ruficapilla (Vieillot, 1819) & $1,33^{\mathrm{a}}$ & $1,13^{\mathrm{a}}$ & $0,50^{\mathrm{b}}$ & $0,03^{\mathrm{c}}$ & $0,03^{\mathrm{c}}$ \\
\hline Synallaxis cinerascens $($ Temminck, 1823) & - & $0,27^{\mathrm{a}}$ & $0,03^{\mathrm{b}}$ & - & - \\
\hline Cranioleuca obsoleta (Reichenbach, 1853) & $0,37^{\mathrm{a}}$ & $0,33^{\mathrm{a}}$ & $0,10^{\mathrm{b}}$ & - & - \\
\hline Automolus leucophthalmus (Wied, 1821) & $0,03^{\mathrm{a}}$ & $0,40^{\mathrm{b}}$ & $0,40^{\mathrm{b}}$ & - & - \\
\hline Heliobletus contaminatus (Berlepsch, 1885) & $0,03^{\mathrm{a}}$ & $0,03^{\mathrm{a}}$ & - & - & - \\
\hline \multicolumn{6}{|l|}{ TYRANNIDAE } \\
\hline Corythopis delalandi (Lesson, 1830) & - & - & $0,37^{\mathrm{a}}$ & $0,13^{\mathrm{b}}$ & $0,10^{\mathrm{b}}$ \\
\hline Hemitriccus diops (Temminck, 1822) & $0,63^{\mathrm{a}}$ & $0,97^{\mathrm{b}}$ & $0,47^{\mathrm{a}}$ & - & - \\
\hline Myiornis auricularis (Vieillot, 1818) & $0,40^{\mathrm{a}}$ & $0,03^{\mathrm{b}}$ & $0,23^{\mathrm{c}}$ & $0,17^{\mathrm{c}}$ & $0,20^{\mathrm{c}}$ \\
\hline Poecilotriccus plumbeiceps (Lafresnaye, 1846) & $1,57^{\mathrm{a}}$ & $0,77^{\mathrm{b}}$ & $0,97^{\mathrm{b}}$ & - & - \\
\hline Capsiempis flaveola (Lichtenstein, 1823) & $1,03^{\mathrm{a}}$ & $0,63^{\mathrm{b}}$ & $0,33^{\mathrm{c}}$ & - & - \\
\hline Platyrinchus mystaceus (Vieillot, 1818) & - & - & 0,07 & - & - \\
\hline Lathrotriccus euleri (Cabanis, 1868) & $0,17^{\mathrm{a}}$ & $0,43^{\mathrm{b}}$ & $0,57^{\mathrm{b}}$ & $0,13^{\mathrm{a}}$ & - \\
\hline Cnemotriccus fuscatus (Wied, 1831) & - & - & 0,03 & - & - \\
\hline
\end{tabular}




\section{Discussão}

Os dados obtidos sugerem que há maior abundância das espécies de aves associadas a bambu onde há maior disponibilidade desse recurso vegetal, o que confirma os dados de outros estudos, como aqueles realizados por Kratter (1997) e Reid et al. (2004). De fato, na trilha $\mathrm{CB} 1$, onde ocorreu maior disponibilidade de bambu, houve maior número de espécies de aves com maior abundância (11 espécies no total). Kratter (1997) já havia verificado esta tendência em uma reserva florestal na região do rio Tambopata, sudeste da Amazônia peruana. Reid et al. (2004), analisando cinco rinocriptídeos especialistas em bambu nas florestas temperadas do Chile encontrou uma correlação positiva entre a abundância destas espécies e a porcentagem de cobertura de bambu, sendo quatro delas mais abundantes nas áreas com maior densidade de bambu. No presente estudo, várias espécies de aves, como Mackenziaena severa, Drymophila malura, Synallaxis rufficapilla, Cranioleuca obsoleta e Capsiempsis flaveola apresentaram clara tendência a um aumento de abundância (IPA) da trilha com menor disponibilidade de bambu (CB3) para a de maior disponibilidade deste recurso (CB1) (Tabela 1).

As espécies de aves que apresentaram maior valor de IPA em CB2 têm sido mais frequentemente associadas a agrupamentos de bambu do interior de floresta, como Drymophila rubricollis, Conopophaga lineata, Hylopezus nattereri, Synallaxis cinerascens e Hemitriccus diops, de acordo com os estudos de Goerck (1999), Leme (2001), Zimmer \& Isler (2003), Fitzpatrick (2004), Volpato et al. (2006) e Antunes (2007). Destas espécies, apenas H. nattereri e C. lineata apresentaram IPA similar nas trilhas CB1 e CB2; H. diops e $S$. cinerascens apresentaram valor de IPA maior na trilha $\mathrm{CB} 2 \mathrm{em}$ relação às outras duas e $D$. rubricollis foi exclusivamente registrada nessa última trilha. $\mathrm{O}$ agrupamento de bambu presente na trilha $\mathrm{CB} 1$ encontrava-se em uma clareira de crescimento secundário, enquanto em CB2 os bambuzais apareceram associados às demais espécies vegetais, numa condição semelhante àquela dos agrupamentos de bambu presentes no interior de floresta. A trilha CB3 foi similar à $\mathrm{CB} 2$ por não ser resultado de crescimento secundário, porém a disponibilidade menor de bambu acabou favorecendo espécies documentadas como não estritamente associadas a bambu, a exemplo de Corythopis delalandi, Platyrinchus mystaceus, Lathrotriccus euleri e Cnemotriccus fuscatus (Fitzpatrick 2004). Assim, CB1, por ser de crescimento secundário, e CB3, por apresentar menor disponibilidade de bambu, tenderam a favorecer espécies de aves oportunistas.

O PG apresenta duas regiões bem distintas, as quais podem ser caracterizadas pela ausência e presença de agrupamentos de bambu. Anjos et al. (2007b) demonstraram que há um predomínio de espécies da guilda de bambu e emaranhados nas áreas correspondentes à CB1 e CB3, localizadas na floresta de encosta do PG, o que foi corroborado pelo presente estudo. Reid et al. (2004) sugeriram a preferência de aves associadas pelo uso da cobertura de bambu como refúgio, evidenciando a importância desse microhábitat como local para abrigo e nidificação. Este autor mostrou uma maior abundância de invertebrados em locais com maiores densidades de Chusquea valdiviensis no sub-bosque da floresta. Ainda, Root (1973) e Frederickson \& Gordon (2003) documentaram uma maior taxa de herbivoria por insetos em agrupamentos vegetais monotípicos. Assim, a maior abundância de aves associadas a esse tipo de ambiente pode estar ligada à maior disponibilidade de recursos alimentares e à proteção que o mesmo pode oferecer.

Apesar de diferenças nos critérios de determinação do grau de associação das aves a bambu, todos os trabalhos que enfocam esse tipo de relação evidenciam a importância e representatividade desse grupo de espécies vegetais na composição de avifaunas regionais. No PG, as espécies de aves associadas ao bambu representam aproximadamente 5\% da avifauna local (Anjos et al. 1997). Kratter (1997), na Amazônia, registrou 32 espécies associadas a bambu que representavam cerca de $7 \%$ da comunidade de aves local. Na Mata Atlântica do sudeste do Brasil, estudos chegaram a identificar até 19 espécies de aves associadas a bambu em um remanescente com menos de 50 ha (Goerck 1999), sendo que Parker et al. (1996) listaram 26 espécies relacionadas a esse microhábitat no bioma em questão. Portanto, unidades de conservação como o PG, que englobam agrupamentos de bambu, podem ser importantes para a conservação de várias espécies de aves, como as analisadas no presente estudo.

\section{Agradecimentos}

O auxílio financeiro para este estudo foi fornecido pela Capes e pelo $\mathrm{CNPq}$ por meio do Programa Mata Atlântica (processo 690146/01-9) e de bolsas de Mestrado a C. R. Santana (processo 133549/2007-0) e Produtividade em Pesquisa a L. dos Anjos (processo 305593/2007-2). Os autores agradecem à Universidade Estadual de Londrina pelo apoio logístico e ao Instituto Ambiental do Paraná pela autorização do estudo nas unidades de conservação do estado. Agradecem também a E.V. Lopes, J. Goerck, L.B. Mendonça, M. Moura e O. Shibatta pelas valiosas contribuições para este manuscrito.

\section{Referências Bibliográficas}

ALEIXO, A., WHITNEY, B.M. \& OREN, D.C. 2000. Range extensions of birds in southeastern Amazonia. Wilson Bull. 112(1):137-142.

ANTUNES, A.Z. 2007. Riqueza e dinâmica de aves endêmicas da Mata Atlântica em um fragmento de floresta estacional semidecidual no sudeste do Brasil. Rev. Bras. Ornitol. 15(1):61-68.

BIANCHINI, E., PIMENTA, J.A. \& DOS SANTOS, F.A.M. 2001. Spatial and temporal variation in the canopy cover in a tropical semi-deciduous forest. Braz. Arch. Biol. Technol. 44(3):269-276.

BLONDEL, J., FERRY, C. \& FROCHOT, B. 1970. La méthode des indices ponctuels d'abundance (IPA) ou des relevés d'avifaune par "stations d'écoute". Alauda 38(1):55-71.

BODRATI, A. \& COCKLE, K. 2006. Habitat, distribution, and conservation of Atlantic forest birds in Argentina: notes on nine rare or threatened species. Orn. Neotrop. 17(2):243-258.

Comitê Brasileiro de Registros Ornitológicos - CBRO (online). 2009. Listas das aves do Brasil. Versão 9/8/2009. http://www.cbro.org.br (último acesso em 22/03/2010).

DOS ANJOS, L. 2007. A eficiência do método de amostragem por pontos de escuta na avaliação da riqueza de aves. Ararajuba. Rev. Bras. Ornitol. 15(2):239-243.

DOS ANJOS, L., VOLPATO, G.H., LOPES, E.V., SERAFINI, P.P., POLETTO, F. \& ALEIXO, A. 2007. The importance of riparian forest for the maintenance of bird species richness in an Atlantic Forest remnant, southern Brazil. Rev. Bras. Zool. 24(4):1078-1086.

FITZPATRICK, J.W. 2004. Family Tyrannidae (Tyrant-flycatchers). In Handbook of the Birds of the World (J. del Hoyo, A. Elliot \& D.A Christie, eds.). Cotingas to Pipits and Wagtails, Barcelona, v. 9, p. $170-257$

FOWLER, J. \& COHEN, L. 1995. Statistics for ornithologists. 2 ed. British Trust for Ornithology Guide 22, Norwich, 150p.

FREDERICKSON, M.E. \& GORDON, D. 2007. The devil to pay: the cost of mutualism with Myrmelachista schumanni ants in 'devil's gardens' is increased herbivory on Duroia hirsuta trees. Proc. R. Soc. Lond., Ser. B: Biol. Sci. 274(1613):1117-1123.

GOERCK, J.M. 1999. Ecology, evolution, and biogeography of Drymophila antbirds (Thamnophilidae, Aves) in the neotropics. Tese de doutorado, University of Missouri, St. Louis, USA. 
JAKSIC, F.M. \& LIMA, M. 2003. Myths and facts about ratadas: bamboo blooms, rainfall peaks and rodent outbreaks in South America. Austral Ecol. 28(3):237-251.

JANZEN, D.H. 1976. Why bamboos wait so long to flower. Annu. Rev. Ecol. Syst. 7:347-391.

JUDZIEWICZ, E.J., CLARK, L.G., LONDONO, X. \& STERN, M.J. 1999. American Bamboos. Smithsonian Institution Press, Washington D.C., 392p.

KRATTER, A.W. \& PARKER III, T.A. 1997. Relantionship of two bamboospecialized foliage gleaners: Automolus dorsalis and Anabazenops fuscus (Furnariidae). Ornithol. Monograph. 48:383-397.

KRATTER, A.W. 1997. Bamboo specialization by Amazonian birds. Biotropica 29(1):100-110.

LEME, A. 2001. Foraging patterns and resource use in four sympatric species of antwrens. J. Field Ornithol. 72(2):221-227.

LLOYD, H. 2004. Habitat and population estimates of some threatened lowland forest bird species in Tambopata, south-east Peru. Bird Conserv. Int. 14(4):261-277.

LOPES, E.V., VOLPATO, G.H., MENDONÇA, L.B., FÁVARO, F.L. \& DOS ANJOS, L. 2006. Abundância, microhabitat e repartição ecológica de Papa-formigas (Thamnophilidae, Passeriformes) na bacia hidrográfica do rio Tibagi, Paraná Brasil. Rev. Bras. Zool. 23(2):395-403.

OHRNBERGER, D. 1999. The Bamboos of the World. Elsevier, Amsterdam, 596p.

OLMOS, F. 1996. Satiation or deception?: Mast-seeding Chusquea bamboos, birds and rats in the Atlantic forest. Rev. Bras. Biol. 56(2):391-401.

OLMOS, F., GALETTI, M., PASCHOAL, M. \& MENDES, S.L. 1993. Habits of the southern Bamboo Rat, Kannabateomys amblyonyx (Rodentia, Echimyidae) in Southeastern Brazil. Mammalia 57(3):325-335.

PARKER III, T.A. 1982. Observations of some unusual rainforest and marsh birds in southeastern Peru. Wilson Bull. 94(4):477-493.

PARKER III, T.A., STOTZ, D.F. \& FITZPATRICK, J.W. 1996. Ecological and distributional databases. In Neotropical birds: ecology and conservation (D.F. Stotz, Fitzpatrick J.W., T.A. Parker III \& D.K. Moskovits, eds.). University of Chicago Press, Chicago, p. 113-436.

PARKER III, T.A., STOTZ, D.F. \& FITZPATRICK, J.W. 1997. Notes on avian bamboo specialists in southwestern amazonian Brazil. Ornithol. Monograph. 48:543-547.

PIERPOINT, N. \& FITZPATRICK, J.W. 1983. Specific status and behavior of Cymbilaimus sanctaemarie, the bamboo antshrike, from southwestern amazonia. Auk 100(3):645-652.
RAJÃO, H. \& CERQUEIRA, R. 2006. Distribuição altitudinal e simpatria das aves do gênero Drymophila Swainson 1824 (Passeriformes: Thamnophilidae) na Mata Atlântica. Rev. Bras. Zool. 23(3):597-607.

REID, S., DIAZ, I.A., ARMESTO, J.J. \& WILLSON, M.F. 2004. Importance of native bamboo for understory birds in Chilean temperate forests. Auk 121(2):515-525.

RODRIGUES, M., ALVARES, S.M.R. \& MACHADO, C.G. 1994. Foraging behavior of the White-collared Foliage-gleaner (Anabazenops fuscus), a bamboo specialist. Orn. Neotrop. 5(1):65-67.

ROOT, R.B. 1973. Organization of a plant-arthropod association in simple and diverse habitats: the fauna of collards (Brassica oleracea). Ecol. Monogr. 43(1):95-104.

SILVEIRA, M. 1999. Ecological aspects of bamboo-dominated forest in southwestern Amazonia: an ethnoscience perspective. Ecotropica 5:213-216.

STOTZ, D.F., FITZPATRICK, J.W., PARKER III, T.A. \& MOSKOVITS, D.K. 1996. Neotropical birds: Ecology and Conservation. University of Chicago Press, Chicago, USA, 502p.

VASCONCELOS, M.F., VASCONCELOS, A.P., VIANA, P.L., PALÚ, L. \& SILVA, J.F. 2005. Observações sobre aves granívoras (Colombidae e Emberezidae) associadas à frutificação de taquaras (Poaceae, Bambusoideae) na porção meridional de Cadeia do Espinhaço, Minas Gerais, Brasil. Lundiana 6(1):75-77.

VEBLEN, T.T. 1982. Growth patterns of Chusquea bamboos in the understory of Chilean Nothofagus forests and their influences in forest dynamics. Bull. Torrey Bot. Club 109(4):474-487.

VIELLIARD, J.E.M. \& SILVA, W.R. 1990. Nova metodologia de levantamento quantitativo da avifauna e primeiros resultados no interior do Estado de São Paulo, Brasil. In Anais do IV Encontro Nacional dos Anilhadores de Aves. Universidade Federal Rural de Pernambuco, Recife, p. 117-151.

VOLPATO, G.H., DOS ANJOS, L., POLETTO, F., SERAFINI, P.P., LOPES, E.V. \& FÁVARO, F.L. 2006. Terrestrial passerines in an Atlantic forest remnant of Southern Brazil. Braz. J. Biol. 66(2a):473-478.

ZIMMER, K.J. \& ISLER, M.L. 2003. Family Thamnophilidae (Typical antbirds). In Handbook of the Birds of the World (J. Hoyo, A. Elliot \& D.A. Christie, eds.). Broadbills to Tapaculos, Barcelona, v. 8, p. 448-681.

Recebido em 08/10/09

Versão reformulada recebida em 26/03/10

Publicado em 09/04/10 